\title{
Balkanologie
}

Balkanologie Revue d'études pluridisciplinaires

Vol. VIII, $n^{\circ} 2$ | 2004

Volume VIII Numéro 2

\section{Revue d'Études Comparatives Est-Ouest, « Sortir de la transition bloquée : Serbie-Monténégro » [numéro spécial]}

Coordinateurs : Mirjana Morokvasic, Nebojša Vukadinović, 35 (1-2), mars-juin 2004, 389 pages

Joseph Krulic

\section{CpenEdition}

\section{Journals}

Édition électronique

URL : http://journals.openedition.org/balkanologie/2070

DOI : $10.4000 /$ balkanologie.2070

ISSN : 1965-0582

\section{Éditeur}

Association française d'études sur les Balkans (Afebalk)

Édition imprimée

Date de publication : 1 décembre 2004

ISSN : 1279-7952

\section{Référence électronique}

Joseph Krulic, "Revue d'Études Comparatives Est-Ouest, « Sortir de la transition bloquée : Serbie-

Monténégro » [numéro spécial] », Balkanologie [En ligne], Vol. VIII, n² 2 | 2004, mis en ligne le 21 janvier 2010, consulté le 17 décembre 2020. URL : http://journals.openedition.org/balkanologie/2070 ; DOI : https://doi.org/10.4000/balkanologie.2070

Ce document a été généré automatiquement le 17 décembre 2020.

(c) Tous droits réservés 


\section{Revue d'Études Comparatives Est-Ouest, "Sortir de la transition bloquée : Serbie-Monténégro » [numéro spécial]}

Coordinateurs : Mirjana Morokvasic, Nebojša Vukadinović, 35 (1-2), mars-juin 2004, 389 pages

Joseph Krulic

\section{RÉFÉRENCE}

Revue d'Études Comparatives Est-Ouest, « Sortir de la transition bloquée : SerbieMonténégro » [numéro spécial], coordinateurs : Mirjana Morokvasic, Nebojša Vukadinović, 35 (1-2), mars-juin 2004, 389 p.

1 Chacun connaît la tradition de recherche approfondie et exigeante de cette revue, interdisciplinaire, mais surtout ouverte vers l'économie. Ce numéro spécial est consacré à la nouvelle « communauté d'États de Serbie-Montenegro » (državna zajedinca Srbija i Crna Gora), née d'un accord constitutionnel en date du 14 mars 2002, entré en vigueur le 3 février 2003. Cette union est localement souvent appelée par dérision «Solania» du nom de Javier Solana, responsable de la politique extérieure de la commission européenne, qui a poussé fermement, avantages économiques à la clé, à cette union provisoire, dont la pérennité, au-delà de 2006, demeure en balance.

Sur ce dernier point, c'est-à-dire l'évolution possible du Monténégro, Amaël Cataruzza montre toute la subtilité et la complexité de «la redéfinition du nationalisme monténégrin dans les crises yougoslaves", évolution marquée, en fin de période (2001-2003), à la fois par une polarisation des identités perçues - au recensement de novembre 2003, seuls 40,64\% des individus recensés se déclaraient « Monténégrins » et 30,01\% «Serbes », alors qu'en 1991, 61,86 \% se déclaraient « Monténégrins » et 9,43\% 
«Serbes» - et par un processus sociologique redoublé d'une polarisation géographique: dans les communes du nord, entre 50,15\% (à Zabjak) et 65,08\% (à Andrijevica) se déclarent "Serbes ", alors qu'au sud, entre 90,23\% (à Cetinje, capitale historique) et $54,94 \%$ à Podgorica (la capitale actuelle, depuis 1945) se déclarent "Monténégrins ». La pression de la «communauté internationale", gênée par la perspective d'un « effet domino » sur le Kosovo voisin d'une éventuelle indépendance monténégrine et l'instabilité des alliances entre partis monténégrins depuis la chute de Milošević, le 5 octobre 2000, rend difficile cependant de prévoir les évolutions futures : une indépendance monténégrine reste possible, sans être certaine.

La première originalité de ce volume est de vouloir lier évolution politique et évolution économique. Cela suppose une prise en compte sérieuse de l'économie. Catherine Samary s'efforce de "réinsérer la Serbie dans l'analyse de la transition ». Elle se livre, notamment, à une comparaison des évolutions socio-économiques de la Serbie et de la Slovénie : en Slovénie, $70 \%$ du « capital social » - contre $60 \%$ en Serbie sous Milošević ont été "vendus» en fonction de "priorités sociales», ce qui montre la réticence, Slovène comme serbe, aux formes affirmées du libéralisme, que certains auteurs appelleraient «le capitalisme anglo-saxon » ou «l'ultra-libéralisme ». La pression de l'Union européenne, dans la perspective d'une intégration, ainsi que la chute de Milošević poussent à l'accentuation de la prédominance des critères libéraux comme bornes de référence. L'analyse de Catherine Samary déborde l'économie, et oppose classiquement - la conception civique à la conception ethnique de la nation, mais cette opposition, admise par certains spécialistes comme Dominique Schnapper et l'américain Roger Brubakers, est relativisée par d'autres auteurs (Alain Renaut, Alain Dieckoff ${ }^{0}$, ou Patrick Weil ${ }^{\circ}$ ). Cela suggère, en tout cas, l'idée que l'originalité de la Serbie ne fut pas celle d'un système économique mais celle d'une situation ou d'une conjoncture, inséparablement politique et économique, déterminée cependant par un choix politique de la Serbie d'isolement (prix de la guerre) qui a conduit la " communauté internationale ", Juridiquement représentée par le Conseil de sécurité de l'ONU, à instaurer des sanctions par la résolution du 30 mai 1992. Comme le montre Michel Drouet dans sa contribution sur « Serbie : quelle transition économique après une décennie de sanctions et de guerre ", les conséquences cumulées de la guerre et des sanctions internationales (chute dramatique du produit national, des revenus, hyperinflation de 1993 à 1000 milliards de \%) se sont traduites par une dégradation du capital investi, préoccupante pour une croissance future. Mais cette situation a accéléré un mal déjà plus ancien, favorisé par une "culture de l'illégalité » et de non-respect des règles et des contrats (p. 95). Les exemples cités par Michel Drouet sont, certes, fort connus : boycott de décembre 1989 des produits Slovènes, émission monétaire non autorisée par la Banque centrale fédérale en janvier 1990, création de pyramides financières comme Dafiment et Jugoskandik à partir de 1992, gel de l'épargne et des devises des ménages et, surtout, l'hyper-inflation de 1992-1993 conduisant, avec l'appauvrissement massif de la population, et notamment de la classe moyenne, à un «transfert de ressources masqué en faveur de l'État ». Les réformes initiales de 2001, rapides, n'ont abouti qu'à « des résultats macro-économiques médiocres » (p. 101). Le rôle des investissements directs étrangers (IDE) se heurte à des risques importants du fait des contingences économiques " ouvrant la voie aux incertitudes et à la corruption », dont Michel Drouet cite un exemple (escroquerie sur le sucre livré par l'UE), alors que « l'appareil judiciaire est peu remanié » et que «l'oligarchie financière » des années 1990 n'a pas été remise en cause. Pour un historien, ce type d'analyse conduit à des interrogations de fonds: 
rôle du passé de longue durée, articulation du culturel et de l'économique dans le lien de causalité de l'évolution générale, économique ou politique. Les noms de Max Weber, de Norbert Elias ne sont pas cités, pas plus que ceux de Fernand Braudel, de l'historien de l'économie Maurice Lévy-Leboyer, spécialiste de l'investissement et de l'industrialisation comparée de la France et de l'Angleterre ou de Joseph Schumpeter. Certes, d'autres noms de classiques viennent à l'esprit, dont Karl Marx, mais l'évocation du rôle des investissements, de la culture du rapport au droit, de la sécurité des contrats ou des escroqueries oblige, notamment, à s'interroger sur l'anthropologie du droit (voir l'œuvre de Norbert Rouland), l'acculturation au droit (voir l'historien médiéviste russe Aaron Gourevitch ${ }^{0}$ et le médiéviste français Alain Boureau). Pour prendre un exemple caricatural, un investisseur britannique, habitué à un monde normatif marqué par huit siècles de Common Law ${ }^{0}$ et plusieurs décennies de droit communautaire, hésitera, toutes choses égales par ailleurs, à se risquer à investir dans un pays où les contrats sont réputés peu sûrs. Entendons-nous : ce raisonnement force le trait de l'idéal-type weberien ou de la caricature ; les contrefaçons, la corruption massive et un rapport au droit à l'opposé de la pratique anglo-saxonne n'empêchent pas des dizaines de milliards de dollars d'investissements de s'engouffrer chaque année en Chine qui connaît, depuis 1978, la croissance que l'on sait. Toutes proportions gardées (mais les proportions ont leur importance : le marché serbe est, du point de vue démographique, 120 ou 130 fois plus restreint que le marché chinois), un jour, la Serbie se réveillera. Ce jour est-il venu? Au regard de l'évolution politique, nous avions un point de vue optimiste sur l'évolution serbe. À lire Michel Drouet, le pessimisme nous gagne. Cela signifie qu'en effet un culturalisme étroit connait vite ses limites : des contextes politiques culturels fort différents ont permis le développement et la croissance économique. Nous serions tentés d'en conclure que la grande croissance serbe est pour bientôt. Mais inversement, l'économisme sans rivage ne permet pas d'expliquer les évolutions particulières; l'économie trouve ses formes spécifiques suivant le rivage qu'elle aborde : la Serbie de 2003 connait, on l'admettra, quelques différences d'avec les Pays-Bas de 1648.

Dans cette conjoncture, les relations avec l'UE et l'aide internationale directe peuvent jouer un rôle déterminant. Daniela Heimerl rappelle qu'après la politique d'isolement et la guerre du Kosovo, le Pacte de stabilité européen de l'été 1999 a voulu inciter les pays de la région à coopérer sur le plan bilatéral et multilatéral. L'exclusion initiale de la Serbie, critiquée par l'opposition, n'a plus lieu d'être après le 5 octobre 2000 et le sommet de Zagreb des 23-24 novembre 2000 a ouvert, en théorie, la perspective d'une adhésion de la Serbie à l'UE, sous la réserve, certes problématique, de remplir les critères : pour Zoran Djindjić, l'horizon 2010 comme date d'adhésion semblait possible. Mais les incertitudes, économiques comme politiques, sur le sort de l'union SerbieMonténégro, et plus encore sur le sort du Kosovo, risquent de reporter l'échéance. À cet égard, l'aide internationale directe, qu'analyse Nebojša Vukadinović, n'a pu être aussi importante que ne l'avaient envisagé les acteurs locaux. La première conférence des donateurs à la zone Serbie-Monténégro, à Bruxelles le 29 juin 2001, n’a pu réunir qu'une somme d'1,279 milliard d'euros au lieu des 3,9 milliards souhaités. Les principaux programmes européens sont les programmes PHARE, OBNOVA et, surtout, CARDS (Community Assistance for Reconstruction, Development and Stabilisation) lequel, créé en décembre 2000 pour les "Balkans occidentaux» - qui recouvrent notamment la Bosnie-Herzégovine - est doté d'un programme de 4,6 milliards d'euros pour la période 2000-2006. L'impact de l'aide semble donc limité pour la Serbie-Monténégro. La seconde 
conférence des donateurs du 18 novembre 2003 a constaté qu'une somme d'1,8 milliard de dollars avait été versée. Mais la structure de l'aide internationale a permis de constater une baisse progressive de dons par rapport aux prêts. Du point de vue sectoriel, l'originalité de l'aide est le soutien direct au budget de l'État, ce qui élève ce pays - c'est implicite dans cette étude - au rang de pays aidés comme l'Egypte, Israël (par les États-Unis d'Amérique) ou les pays d'Afrique francophone (par la France). Spontanément, du fait de la baisse de l'apport des privatisations et des rentrées fiscales incertaines dues à un PIB encore limité ou dont la croissance alimente une économie souterraine, le déficit budgétaire croît.

Dans ce recueil, on peut également lire d'excellentes analyses ou synthèses sur l'évolution politique de la Serbie-Monténégro (articles de Diane-Masson sur les « freins au processus de démocratisation en Serbie », d'Yves Tomic " une vie politique en Serbie de 1987 à 2004 : une chronologie »), mais la véritable originalité de ce numéro est d'essayer d'articuler non seulement évolution politique et évolution économique, mais aussi évolution sociale, théorie générale de science politique ou de sociologie politique (voir l'article sur «les obstacles sociaux et institutionnels au processus de transformation en Serbie » de Mladen Lazić, sociologue à l'université de Belgrade) et, dans une mesure certes très limitée, évolution juridique. À cet égard, l'article de Marina Glamotchak sur l'« établissement de l'État de droit et le contrôle des unités spéciales de la police " qui porte, en réalité, sur l'organisation des unités spéciales de sécurité policières, le rôle en creux de l'armée, la répression après l'assassinat du Premier ministre Zoran Djindjić, le 12 mars 2003, restera comme une précieuse contribution sur les évolutions des structures du système policier serbe de 1990 à 2003. Mais, mis à part deux allusions (la visite de Caria del Ponte à Belgrade [p. 338] et les réticences, juridiquement fondées dans le droit interne serbe, des unités de sécurité serbes pour coopérer avec le TPIY pp. 340-341]) et quelques lignes sur les "purges au sein de l'appareil judiciaire» (pp.345-346), lesquelles demeurent un projet inaccompli des nouveaux gouvernements serbes (35 juges licenciés en 2003, peut-être trop indépendants aux yeux dudit gouvernement), rien n'est dit ni sur l'État de droit dont la définition, depuis Hans Kelsen et René Carré de Malberg, suppose l'examen de la hiérarchie des normes juridiques internes d'un État, du respect (contrôlé par une pyramide de juges) de chaque niveau de normes inférieures au regard des normes supérieures (normes réglementaires au regard des normes législatives, normes législatives au regard des normes constitutionnelles), ni sur la question de l'articulation du droit interne au regard du droit international, tant le droit international public que le droit international pénal en voie de constitution (au minimum depuis le statut du tribunal de Nuremberg du 9 août 1945). Le TPIY pose au système juridique serbe comme à bien d'autres d'ailleurs, notamment, dans la région, la Croatie - de redoutables problèmes qui renvoient à une problématique plus générale (voir, par exemple, l'œuvre de la juriste Mireille Delmas-Marty, non citée par les auteurs). À cet égard, et par un paradoxe qui n'est qu'apparent, les unités spéciales de bérets rouges qui, comme le rappelle Marina Glamotchak (p.340), "appellent au respect du constitutionnalisme ", avaient probablement raison, au regard du droit interne serbe ou « serbo-monténégrin ». À supposer qu'ils aient politiquement tort (mais qui peut en décider?), ils avaient, en droit interne, juridiquement raison. Mais avaient-ils juridiquement raison au regard du droit international? Immense problème, qui supposerait une étude dont l'ampleur dépasse notre compte-rendu bibliographique. On peut, au moins, noter que ce problème n'a rien de «balkanique » comme on le croit 
trop souvent. Il suffit de lire les décisions du Conseil constitutionnel français sur la Cour pénale internationale (décision du 29 janvier 1999 déclarant nécessaire une réforme de la Constitution pour adopter le Traité de Rome du 17 juillet 1998 créant la Cour pénale internationale permanente, avec une incise fameuse sur l'immunité pénale du chef de l'état) ou de récentes décisions sur l'articulation entre droit communautaire et contrôle de constitutionnalité, pour avoir un autre regard sur le défunt Zoran Djindjić, la livraison de Slobodan Milošević au TPIY, etc. L'étude des relations entre droit américain et ratification des conventions internationales, les décisions du tribunal constitutionnel de Karlsruhe sur ce que la RFA était autorisée à faire en droit international ou encore de la réception « dualiste » des traités par le droit britannique nous plongerait dans des délices comparables. De même « l'état d'urgence » signalé par Marina Glamotcak, après l'assassinat de Zoran Djindjić du 12 mars 2003, mériterait une étude en soi, au regard d'un hypothétique "État de droit» réel ou virtuel. Est-il conforme au droit serbe? Au droit serbo-monténégrin? Quelles sont ses bases juridiques, réelles ou supposées? Est-il possible de la comparer à d'autres régimes juridiques (PatriotAct américain de 2001, régime français de l'article 16 de la Constitution française) ? La question n'est pas que juridique : des philosophes du droit ou juristes fort divers, classés à droite (Carl Schmitt, rallié juridiquement au régime nazi, du fait, notamment, de sa théorie de la décision et des circonstances exceptionnelles) ou à gauche (l'Italien Giorgio Angabem, opposé au Patriot Act après le 11 septembre 2001 mais théoricien de «l'état d'urgence » comme limite inévitable de tout système juridique) ont éclairé la question, avec des paradoxes qui ouvrent des abîmes : Hans Kelsen, président et fondateur de la Cour constitutionnelle autrichienne en 1920, juif autrichien chassé par le nazisme, se croyait obligé de reconnaître la légalité du régime nazi, au nom de sa théorie positiviste de l'État de droit comme pyramide de normes qui ne trouvent de fondements que dans l'État. Les évolutions serbes de 2002-2003, permettent, notamment, selon nous d'illustrer de semblables débats.

Curieusement, de ce point de vue, Marina Glamotctchak note, p. 346, "par ailleurs, l'appareil judiciaire paraît désormais être le seul responsable de la lutte contre la criminalité organisée, comme si le ministère de l'Intérieur n'était pas concerné ». Mais par définition, l'État de droit (voir supra) ou même la simple légalité dans un État non dictatorial comme l'était la France de la III république selon René Carré de Malberg, suppose le contrôle de juges (initiative du ministère public, juges d'instruction dans une procédure inquisitoriale, juges du siège se prononçant sur la culpabilité éventuelle) sur les policiers. Dans un État d'un autre type, qui tolère des marges de violence, les policiers d'un autre type, officiels ou officieux, officient seuls ou presque : de l'affaire Ben Barka en France en 1965, aux 550 assassinats peu élucidés dans la Serbie de Slobodan Milošević (voir, pour mémoire, Ivan Stambolić, enlevé alors qu'il faisait son jogging et assassiné fin août 2000, événement désormais mieux connu). Une caractéristique de la Serbie-Monténégro, dans les années 1990-2003 fut justement qu'elle a, comme l'Allemagne de Weimar en 1920-1923, pour ne parler que d'un régime pluraliste, toléré l'assassinat politique comme régulateur implicite du système politique, tout en tolérant simultanément, dans tous les sens du mot tolérer, une opposition politique. Cette double tolérance ou double postulation, qui pourrait être éclairée par un comparatisme historique et de sociologie politique, distingue radicalement la Serbie de cette période (au Monténégro, à notre connaissance, les assassinats politiques ne furent pas courants), et singularise radicalement ce pays de 
l'Irak de Saddam Hussein (assassinats nombreux, mais officiels, pas de prétention à l'État de droit ou au pluralisme politique) ou d'autres pays (assassinats politiques rares, avec des tentatives de répression réelles, mais controversées, comme les États-Unis des années 1960). L'assassinat de Zoran Djindjić marque à cet égard un tournant. Il est possible, et c'est notre hypothèse, que les historiens considéreront, un jour, que le changement de régime en Serbie date de cet événement (12 mars 2003). Nous rejoignons par là même le problème de l'État de droit : Zoran Djindjić, né en Bosnie, docteur en philosophie de l'université de Constance (Allemagne), se voulait lecteur d'Habermas dont, en effet, le monumental Droit et Démocratie ${ }^{0}$ constitue une illustration ou une introduction possible du problème de l'État de droit. Ce que la vie de Zoran Djindjić n'a pas réussi à instaurer en Serbie - l'État de droit - sa mort peut-elle contribuer à l'établir? L'on voit ici que les problèmes «balkaniques" renvoient à des problèmes sinon « universels » (?) du moins plus généraux ou « universalisames » pour reprendre un schéma kantien que ne désavoueraient ni Habermas ni, sans doute, Djindjić.

7 À cet égard, le très original et brillant article de Thomas Meekel, spécialiste travaillant au ministère des Finances de Serbie, («Serbie : comment rebâtir la relation entre l'État et les collectivités locales?»), nous plonge au cœur des contradictions politiques, juridiques et financières de ce régime et des évolutions souhaitées par certains depuis 2002. La Yougoslavie de Tito avait largement évolué dans un sens globalement décentralisateur. L'auteur signale la Constitution du 21 février 1974 mais, de manière plus pertinente, on pourrait rappeler - ce que ne fait pas l'auteur - la grande réforme du système des communes de 1957 voulue par Edvard Kardelj, lequel pensait que la commune était la cellule fondamentale de l'État et une pièce essentielle d'une société autogérée. On sait que, dans sa volonté d'en finir avec la Yougoslavie de Tito-Kardelj, supposée avoir minoré le rôle du peuple serbe (notamment, en permettant, l'autonomie du Kosovo et de la Voïvodine), le projet politique de Slobodan Milošević était fondamentalement re-centralisateur. L'opposition à Milošević, fort logiquement, a connu ses premiers succès dans la mobilisation des collectivités locales (victoire dans quatorze communes importantes aux élections municipales du 16 novembre 1996, comme le note aussi Diane Masson, dans une note p. 43, et manifestations populaires devant le refus initial du régime de reconnaître le résultat de ces élections). Le gouvernement Djindjić s'est donc cru obligé de faire une réforme de décentralisation par une nouvelle loi des collectivités locales du 14 février 2002. Mais, comme le savent tous les connaisseurs des problèmes de décentralisation, celle-ci est plus simple à souhaiter qu'à réaliser. La réforme en cause a été précédée de larges consultations avec les collectivités locales de Serbie, ainsi que d'une analyse comparative avec les législations des "pays voisins" et des «tendances des systèmes locaux dans les pays développés ». En effet, comme le note finement l'auteur, la plupart des États issus de l'ex-Yougoslavie ont connu, pendant la crise des années 1990, une phase de centralisation (le cas de la Croatie est éloquent et les problèmes des collectivités locales en Macédoine ou en Slovénie pourraient utilement compléter l'analyse), du fait, pourrions nous ajouter, de la corrélation entre centralisation administrative et légitimité politique incertaine d'un système, qui se perçoit comme menacé par les oppositions internes et des ennemis externes. À cet égard, le contrôle financier des collectivités locales et le contrôle de légalité a priori des mêmes collectivités instaurées par Milošević rappellera au lecteur français un tableau familier: avant la loi de décentralisation française du 2 mars 1982, et en tous cas avant les premières réformes 
de 1964, le schéma de surveillance des collectivités locales par l'État central en France était semblable (contrôle préalable des actes du point de la légalité et des autorisations de paiement, flux financiers régulés par l'administration centrale). Les problèmes rencontrés par la réforme serbe du 14 février 2002 sont également connus sous d'autres cieux : la loi consacre un tiers des 154 articles aux finances locales. Sous Milošević, comme le note l'auteur p. 306, «les recettes des collectivités locales proviennent à hauteur de $70 \%$ à $80 \%$ des transferts du gouvernement central» et les recettes proviennent du partage d'impôts levés par l'administration fiscale de l'État central. La «nouvelle réforme a voulu transférer des recettes stables aux collectivités locales, à compétences restées globalement égales»(p.311), ce qui a doublé le revenu des communes serbes entre 2000 et 2003, lesquelles représentent $6 \%$ du PIB serbe en 2003 et non plus $4 \%$ comme en 2000. Comme le relève une étude spécialisée citée par Thomas Meekkel, la part du PIB consacrée aux collectivités est comparable en Serbie, voire supérieure aux autres pays de l'Europe du sud-est. Si ces réformes demeurent, comme il le relève, fragiles et d'une cohérence douteuse (un exemple : l'établissement de tribunaux administratifs, sur le modèle français ou allemands, prévu en janvier 2003, a été retardé du fait de problèmes financiers; or, c'est le corollaire habituel de l'abandon d'un contrôle de légalité préalable remplacé, en cas de décentralisation véritable, par un contrôle a posteriori des juridictions administratives), l'État central hésite entre un rôle de contrôle direct difficile à maintenir et un rôle de coordination ou de stratège qui reste, en tout état de cause, utile (pour garantir un minimum d'égalité de traitement géographique de traitement pour les services publics ou la péréquation financière). Si le « centralisme reste ancré dans toutes les têtes, y compris celle des élus locaux, très présents à Belgrade, puisque tout s'y décide ", le système de collectivités locales "dispose déjà d'un maillage du territoire cohérent et rationnel » (p.319). Du fait de la réforme titiste de 1957, que l'auteur ne cite pas (voir supra), les communes sont fort peu nombreuses: 160 dans la Serbie au sens strict, Kosovo non compris, soit un ratio de 45000 habitants et $475 \mathrm{~km}^{2}$ en moyenne par commune, avec quatre villes d'une importance nettement supérieure à cette moyenne. Comme le signale l'auteur, la Serbie devrait éviter de suivre la Slovénie qui, dans sa loi du $1^{\mathrm{er}}$ janvier 1995, a remplacé les 62 communes instituées en 1957 par 147 nouvelles communes, nombre porté à 193 en 2002 (rappelons que la France a plus de 35000 communes). Mais de nombreuses réformes techniques, longues et difficiles, sont nécessaires: des outils de gestion, des réformes et une meilleure rémunération des entreprises locales, des prestations (eaux,...), une formation continue des fonctionnaires locaux, etc. On pourrait, toutefois, ajouter, sur ce point comme sur les autres, que ce type de démarche dépasse largement, le cas serbe.

8 En revanche, une certaine spécificité du pays demeure pour ce qui concerne les migrations humaines. Dans l'article de Mirjana Morovkavsic "Serbie-Monténégro: terre de refuge, terre de départ", on voit que dans les années 1990, le nombre important de réfugiés (646 0000 en 1996, 451000 en 2001, 279000 en 2004, sans compter les 228000 « déplacés » du Kosovo recensés en 2001, devenus 206000 en 2004), comme celui des départs (chiffre difficile à évaluer, sans doute supérieur à 300000 ) est largement lié à la conjoncture politique. Selon le recensement de 2002, les réfugiés représentent $5 \%$ de la population totale et près de $10 \%$ de celle de la Voïvodine. Parmi ceux qui quittent le pays, la part des diplômés de l'enseignement supérieur augmente (ils passent de 1,8 \% en 1991 à 6,2 \% en 2001) et, parmi ceux qui sont partis depuis 1991, ceux d'un niveau secondaire et supérieur seraient en nombre quatre fois plus élevé que 
dans la population locale de référence ; le taux d'émigration des chercheurs a, lui, varié entre $4,5 \%$ et $15,3 \%$ selon les instituts. Mais une nouvelle relation, réelle ou virtuelle (Internet aidant) s'instaure entre la "diaspora» et la Serbie-Monténégro: le gouvernement Djindjić a confié trois postes ministériels à des hommes de la diaspora, dont le ministère des Finances au très français Borislav Djelić (HEC, arrivé en France à l'âge de 7 ans, marié à une sociologue française restée à Paris). Les exilés ou réfugiés à l'étranger réclament l'amnistie pour leur fuite devant le service militaire ou pour la perte des biens sous le premier titisme en 1945-1948. Le prince Alexandre Karadjordjević a récupéré le palais Blanc occupé par Tito puis par Milošević, mais d'autres réclament des biens plus modestes. On pourrait ajouter, ce que Mirjana Morokvasic ne précise pas même si elle note que ces revendications veulent rapprocher la Serbie de l'Europe, que la Serbie-Monténégro étant membre du Conseil de l'Europe depuis 2001, ils peuvent se prévaloir de l'article premier du Premier protocole additionnel à la Convention européenne des droits de l'Homme qui protège les biens : cet article permet déjà, par exemple, aux réfugiés serbes de Croatie d'obtenir de la Cour européenne des Droits qu'elle ordonne à la Croatie la restitution de leurs biens ou une indemnisation équivalente. Le gouvernement serbe n'a aucun moyen d'empêcher cette restitution des biens. Du fait de son appartenance au Conseil de l'Europe, la SerbieMonténégro est déjà dans une certaine Europe. Les électeurs de la diaspora qui ont massivement voté pour Boris Tadić lors du second tour de l'élection présidentielle du 27 juin 2004 l'ont compris. On retrouve ici une problématique plus générale : la Serbie, à la fois terre d'accueil de réfugiés et terre de départ de cadres, comme c'est le cas de beaucoup de pays d'Europe (dont la France et le Royaume-Uni), n'est pas une île balkanique, mais une partie de l'Europe.

Ce recueil d'articles sur la Serbie-Monténégro restera un document de référence pour l'analyse de cet État dans les années 1990-2004. Il contribue à "débalkaniser " la problématique de la recherche et le regard qu'une opinion éclairée peut avoir sur cet État. Il sollicite de nombreuses questions, débats et prolongements possibles dans la réflexion et la recherche, notamment comparative. En mettant l'accent sur des points décisifs mais peu étudiés (comme, par exemple, la baisse du stock de capital dans l'article de Michel Drouet), il évitera à beaucoup des contresens dans l'analyse dudit État. Toutefois, dans un souci de correction de l'image "balkanique» du pays, beaucoup d'articles en viennent à ne pas vouloir s'interroger sur d'hypothétiques particularités serbes issues de la très longue durée historique. Cela ne serait pas contradictoire avec la «débalkanisation» du pays. Dire, par exemple, que la centralisation, au moins depuis les années 1630-1660, constitue un phénomène déterminant en France qui la distingue de l'Allemagne ou des États-Unis, n'empêche pas de constater qu'entre les problèmes de décentralisation en France, en Serbie, en Croatie, etc., de très nombreuses similitudes techniques et politiques existent. Cela est vrai pour les problèmes économiques : en France, en Chine, en Serbie, la croissance économique suppose une combinaison de facteurs dont le travail, le capital investi, mais aussi un ensemble de variables plus difficiles à définir (capital humain, sécurité juridique, etc.) qui suppose une évolution spécifique. Le risque d'assassinat, pour un homme politique ou public, n'était certes pas aussi élevé en France, ni même en Macédoine, Slovénie, Croatie ou même Monténégro dans les années 1990-2003, qu'en Serbie et au Kosovo, dans les mêmes années. Il ne s'agit pas de revenir au long débat Balkans/Europe centrale: l'Allemagne de Weimar et les États-Unis des années 1865-1929, comme la Suisse de 1847-1849 ont connu des formes de violence politique, 
incluant l'assassinat. Il s'agit encore moins, selon nous, de songer qu'un « fascisme » du long $\mathrm{xx}^{\mathrm{e}}$ siècle expliquerait ces violences. Sans doute les auteurs qui se sont efforcés de mener des analyses précises sur la Serbie-Monténegro ne pouvaient, l'eûssent-ils voulu et malgré les tentatives de certains (voir l'article déjà cité de Mladen Lazić), se livrer à ce genre de spéculations. Mais le numéro ouvre à un monde de réflexions. C'est l'apport que l'on peut demander à des publications de référence.

\section{NOTES}

0. Voir l'analyse de la philosophie de Fichte de Renaut (Alain), «Logiques de la nation », in Delannoi (Gil), Taguieff (Pierre André), éds., Théories du nationalisme. Nation, nationalité, ethnicite, Paris : Kimé, 1991 ; et Dieckoff (Alain), « Nationalisme politique et nationalisme culturel », l'Année sociologique, 46 (1), 1996.

0. Weil (Patrick), Qu'est-ce qu'un Français? Histoire de la nationalité française depuis la Révolution, Paris : Grasset, 2002.

0. Gourevitch (Aaron) Les catégories de la culture médiévale, Paris : Gallimard, 1983, chapitre « Un pays se construit sur le droit », pp. 157-211

0. Voir Boureau (Alain), La loi du Royaume, les moines, le droit et la construction de la nation anglaise (XI-XII ${ }^{\text {éme }}$ siècles), Paris : Les Belles-Lettres, 2001.

0. Habermas (Jurgen), Démocratie et droit, entre faits et normes, Paris : Gallimard, 1997. 